\title{
Empfehlungen zur Verwendung dieses Buches
}

Der Schritt von der soeben beendeten Schule in ein technisches Studium ist meist ein sehr großer. Während in der Schule eine breitere Wissensvermittlung im Vordergrund stand, spezialisiert man sich jetzt auf ein ganz bestimmtes Fachgebiet, um später einen entsprechenden Beruf ausüben zu können. Auch das Studium selbst hat Ähnlichkeiten zum Berufsalltag. Anders als in der Schule ist man wesentlich mehr verpflichtet, sich um das eigene Bestehen im Studium zu kümmern. Prüfungen gibt es nur einmal im Semester, und die müssen bestanden werden, da man sonst sehr schnell auf einen Schlingerkurs gerät. Eine der größten Schwierigkeiten wird die Motivation sein, immer wieder Neues zu lernen, auch wenn es anstrengend ist. Mathematik und Physik sind in den technischen Studiengängen deswegen prädestiniert, bei Studenten für Unmut zu sorgen. Die Vorlesungen schreiten oft sehr schnell voran, der Stoff wird aus Zeitgründen komprimiert vermittelt. Gedankengänge nachzuvollziehen erfordert ein großes Maß an Konzentration und der entstehende Frust bei ausbleibendem Verständnis treibt die Motivation schnell in den Keller. Auch die Autoren dieses Buches haben diese Erfahrung gemacht. Bei der Konzeption stand deswegen die Ausführlichkeit des Lehrstoffs im Vordergrund. Der Leser soll die Möglichkeit haben, auch abseits exakter mathematischer Formulierungen durch das Lesen von reinem Text ein Verständnis zu erlangen. Oft sind Gedankengänge, die sich in einer Abfolge von Gleichungen niederschlagen, davor in Worte zusammengefasst. Formeln quantifizieren die vorangestellten Überlegungen, indem sie physikalische Größen (abgekürzt durch Formelzeichen) in einen Zusammenhang stellen, der zahlenmäßige Aussagen erlaubt. Der Leser wird feststellen, dass das Lernen in der Physik deutlich leichter wird (und im besten Fall sogar Spaß macht), wenn er den roten Faden nicht verliert. Wie sich dieser durch die einzelnen Kapitel zieht, wird durch kurze Rückblicke im Text verdeutlicht.

Herleitungen von wichtigen Ergebnissen erfolgen ohne die Verwendung von Zahlen, da diese keine Möglichkeit bieten, Zusammenhänge zu verstehen. So wird man durch bloßes Verrechnen von Zahlenwerten beispielsweise immer wieder feststellen, dass man Raketen unterschiedlicher Massen immer auf die gleiche Geschwindigkeit bringen muss, damit sie das Gravitationsfeld der Erde verlassen können. Aber erst eine Herleitung der Fluchtgeschwindigkeit zeigt, dass diese von der Raketenmasse nicht abhängt. Dieser Umstieg auf abstraktere Herleitungen ist notwendig, da unsere heutige Technik eine derartige Abstraktion erfordert, um beherrschbar zu bleiben. Man macht sich am besten klar, dass Herleitungen nur Gedankengänge darstellen, die man (allerdings in wesentlich längerer Form) auch in Worte fassen könnte. Es bietet sich sogar an, die verwendeten Buchstaben gedanklich mit den Namen für die verwendeten Größen zu versehen, also nicht $m$ zu lesen, sondern „Masse“. An einigen Stellen findet sich auch im Text eine solche Ausformulierung. Außerdem werden auch in der Praxis Berechnungen am Computer gemacht, denen das Schreiben eines Programms vorausgeht. Dafür gibt es speziell für den Ingenieurbereich angepasste Softwarepake- 
te, und die Denkweise beim Erstellen eines Programms lehnt sich sehr stark an die Formelsprache an, die in der Physik verwendet wird. Zusätzlich wird die Anwendung der erarbeiteten Ergebnisse natürlich durch Beispiele verdeutlicht, um ein Gespür für das Rechnen und auch die Größenordnungen zu erlangen. Auch diese werden sehr ausführlich gerechnet, um einerseits den Gedankengang aufzuzeigen, der für die Lösung eines Problems nötig ist. Schließlich bedeutet „Problemlösung“ nicht, die passende Formel zu suchen und lediglich Zahlen einzusetzen. Andererseits kommt es auch beim Einsetzen von Zahlen oft zu Unfällen, da eine Formel physikalische Größen in einer bestimmten Einheit erwartet. So sollte man z.B. für eine Temperatur von $20^{\circ} \mathrm{C}$ nicht den Zahlenwert 20 in den Taschenrechner eingeben, wenn in der Formel der Wert in der Einheit Kelvin benötigt wird und deswegen die Zahl 293,15 die richtige gewesen wäre (ein Unterschied von immerhin einer ganzen Größenordnung ... ). Die Liste solcher Beispiele ist lang. Doch nicht nur vorgerechnete Beispiele sollen das Einüben ermöglichen, auch an Aufgaben, die jeweils am Ende eines Abschnitts angeboten werden, kann der Leser sein Verständnis und seine Rechenfertigkeiten austesten.

Dieses Buch setzt bei den Kenntnissen an, die in der Schule erlangt wurden und führt dann in den einzelnen Kapiteln jeweils mehr und mehr Konzepte ein, die gegen Ende immer deutlicher über das Schulniveau hinausgehen. Das Umformen von Termen nach einer bestimmten Größe gehört zu den vorausgesetzten mathematischen Kenntnissen, ebenso sollte der Leser wissen, was eine lineare und eine quadratische Funktion ist. Differential- und Integralrechnung gehören zu den anspruchsvollsten Dingen, die im Mathematikunterricht besprochen werden. Wir werden diese im Zuge der Anwendung auf physikalische Probleme noch einmal in neues Licht rücken. Teilweise verzichten wir dabei auf mathematische Exaktheit, um die Anschauung hervorzuheben. Auch die Vektorrechnung werden wir wiederholen. Insbesondere werden es auch Schreibweisen sein, die im Studium vorkommen und die besonders am Anfang zu Problemen führen können. Wir werden dem eine entsprechende Einführung an den jeweiligen Stellen entgegensetzen. Somit sollte der Leser das Buch schon im Vorkurs verwenden können, die komplexeren Teile der einzelnen Kapitel sollen dem eigentlichen Studium vorbehalten sein.

Herleitungen von wichtigen Sachverhalten können sich manchmal etwas hinziehen. Dies ist ein gutes Stück der großen Ausführlichkeit geschuldet, welche zu kleineren, aber auch zahlreicheren Rechenschritten führt. Der Leser soll beim Anblick einer Seite mit vielen Gleichungen deswegen nicht gleich den Mut verlieren. Der beste Weg ist der des Nachvollziehens der einzelnen Schritte, eventuell mit der einen oder anderen kleinen Notiz. Wenn die einzelnen Schritte erst einmal verstanden sind, stellt sich auch ein besseres Verständnis für Zusammenhänge ein.

Wir wünschen einen guten Start in den Vorkursen und ein erfolgreiches sowie spannendes Studium! 